\title{
Optimized, Minimal Specific Absorption Rate MRI for High-Resolution Imaging in Patients with Implanted Deep Brain Stimulation Electrodes
}

\author{
(D) A.M. Franceschi, (D) G.C. Wiggins, (D)A.Y. Mogilner, (D). Shepherd, DS. Chung, and (D).W. Lui
}

\begin{abstract}
BACKGROUND AND PURPOSE: Obtaining high-resolution brain MR imaging in patients with a previously implanted deep brain stimulator has been challenging and avoided by many centers due to safety concerns relating to implantable devices. We present our experience with a practical clinical protocol at 1.5T by using 2 magnet systems capable of achieving presurgical quality imaging in patients undergoing bilateral, staged deep brain stimulator insertion.
\end{abstract}

MATERIALS AND METHODS: Protocol optimization was performed to minimize the specific absorption rate while providing image quality necessary for adequate surgical planning of the second electrode placement. We reviewed MR imaging studies performed with a minimal specific absorption rate protocol in patients with a deep brain stimulator in place at our institution between February 1, 2012, and August 1, 2015. Images were reviewed by a neuroradiologist and a functional neurosurgeon. Image quality was qualitatively graded, and the presence of artifacts was noted.

RESULTS: Twenty-nine patients (22 with Parkinson disease, 6 with dystonia, 1 with essential tremor) were imaged with at least 1 neuromodulation implant in situ. All patients were imaged under general anesthesia. There were 25 subthalamic and 4 globus pallidus implants. Nineteen patients were preoperative for the second stage of bilateral deep brain stimulator placement; 10 patients had bilateral electrodes in situ and were being imaged for other neurologic indications, including lead positioning. No adverse events occurred during or after imaging. Mild device-related local susceptibility artifacts were present in all studies, but they were not judged to affect overall image quality. Minimal aliasing artifacts were seen in 7, and moderate motion, in 4 cases on TIWI only. All preoperative studies were adequate for guidance of a second deep brain stimulator placement.

CONCLUSIONS: An optimized MR imaging protocol that minimizes the specific absorption rate can be used to safely obtain high-quality images in patients with previously implanted deep brain stimulators, and these images are adequate for surgical guidance.

ABBREVIATIONS: $\mathrm{DBS}=$ deep brain stimulator; $\mathrm{RF}=$ radiofrequency; $S A R=$ specific absorption rate

D eep brain stimulation is an effective treatment for medically refractory movement disorders, including Parkinson disease, essential tremor, and dystonia. Imaging plays a critical role in stereotactic targeting and long-term assessment. Preoperative MR images routinely used for deep brain stimulator (DBS) placement guidance include a high-resolution T1-weighted sequence used to identify standard anatomic landmarks such as the anterior/posterior commissures and high-resolution T2-weighted MR imaging routinely used to target the subthalamic nucleus, the

Received November 18, 2015; accepted after revision May 4, 2016.

From the Departments of Radiology (A.M.F., G.C.W., T.S., S.C., Y.W.L.) and Neurosurgery (A.Y.M.), New York University School of Medicine, New York, New York.

Please address correspondence to Yvonne W. Lui, MD, Department of Neuroradiology, 660 First Ave, 2nd Floor, New York, NY 10016-6481; e-mail:

Yvonne.lui@nyumc.org

http://dx.doi.org/10.3174/ajnr.A4865 most common structure targeted in deep brain stimulation for Parkinson disease. For patient safety and to judge treatment efficacy, placing bilateral leads in staged unilateral procedures can be advantageous. Due to the precision required for stereotaxis and the size of the anatomic structures (Fig 1), immediate preoperative imaging is the standard of care. When staged procedures are used, this necessitates imaging with 1 electrode in place for subsequent placement of the second electrode. Additionally, electrode-in imaging may be required to assess lead placement. Manufacturer's guidelines for performing MR imaging with DBSs in situ are extremely conservative; this feature makes acquiring diagnostic and therapeutic imaging in these patients challenging.

The recommended head specific absorption rate (SAR) limit for Medtronic DBS systems (Medtronic, Minneapolis, Minnesota) has been $0.1 \mathrm{~W} / \mathrm{kg}$ (compared with the usual normal mode, 


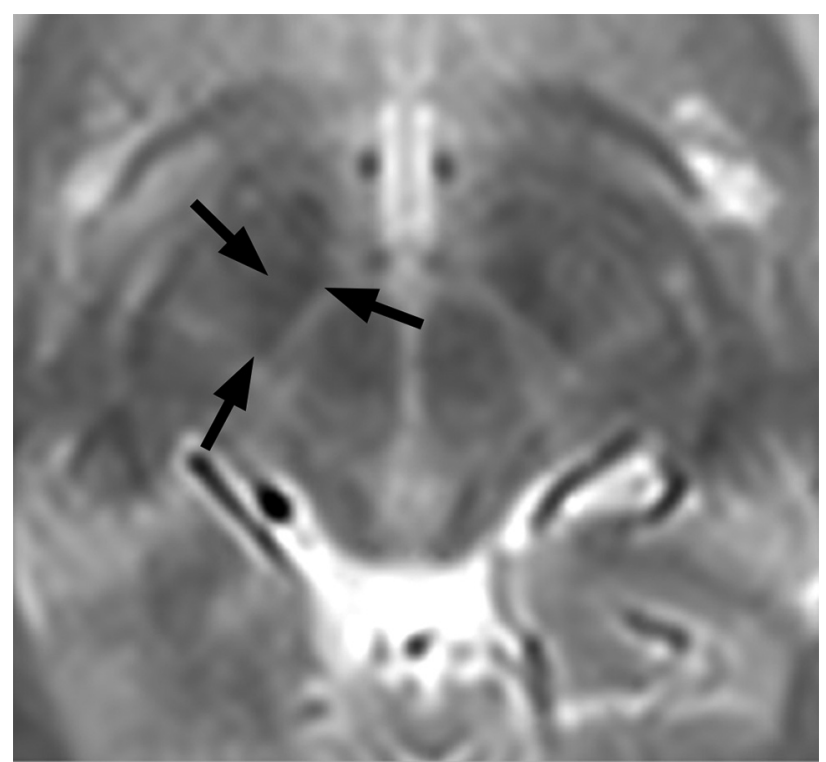

FIG 1. The smallest of the basal ganglia nuclei can be delineated on appropriate $\mathrm{T2}$-weighted imaging through a region just cephalad to the midbrain. The subthalamic nucleus, a frequent target for deep brain stimulation, is outlined by arrows on the patient's right.

which calls for SAR $<3.2 \mathrm{~W} / \mathrm{kg}$ ). These devices are rated conditional at $1.5 \mathrm{~T}$. The main safety concern is heating the electrode due to energy deposition, and a few prior complications have been reported in the literature ${ }^{1,2}$; however, Larson et $\mathrm{al}^{3}$ described 405 patients imaged with implanted DBS systems by using a variety of different scanning protocols, and their review suggests that a head SAR up to $3.0 \mathrm{~W} / \mathrm{kg}$ may be applied without untoward incidents. Other potential interactions between MR imaging and implantable neuromodulators include magnetic field interactions, induced stimulation, effects on neurostimulator function, and artifacts from the device. In a single published article, Sarkar et $\mathrm{al}^{4}$ reported being able to achieve diagnostic quality within the manufacturer's SAR limit with research 3D spin-echo sequences. The research sequences used in that study are not universally available, and the method they used most likely underestimated the loss of the signal-to-noise ratio. There is no clear consensus in the literature as to the optimal SAR and MR imaging parameters for safe imaging of patients with DBS electrodes.

The purpose of this study was to describe our experience with an MR imaging protocol by using product sequences, optimized for both therapeutic image quality in patients with implanted DBS electrodes and low SAR on 1.5T clinical scanners.

\section{MATERIALS AND METHODS}

The study was approved by the institutional review board, and the imaging protocol was approved by the institutional MR imaging safety committee.

\section{Patients}

Patients with neuromodulation devices, including Itrel II Model 7424, Soletra Model 7426, Kinetra Model 7428, Activa PC Model 37601, Activa RC Model 37612, Activa SC Model 37602, Activa SC Model 37603 (Medtronic), referred by the Center for Neuromodulation at the New York University Department of Neurosurgery for
MR imaging, were included in this protocol. Patients with bilateral DBS devices all had separate pacemakers, separated by $6 \mathrm{~cm}$.

\section{Protocol Optimization}

Axial T1-weighted magnetization prepared rapid acquisition of gradient echo is an intrinsically low-SAR sequence and requires little optimization. SAR-limited T2-weighted protocols were constructed by varying TRs, resolutions, flip angles, and radiofrequency (RF) pulse types. Use of the manufacturer-supplied lowSAR RF pulse reduced the SAR to $58 \%$ of the value with the default RF pulse. The target resolution to provide diagnostic information was $1 \mathrm{~mm}$ in-plane with a $2.5-\mathrm{mm}$ section. The applied strategy was to keep TEs and flip angles constant and to reduce the SAR by lowering the number of RF pulses per unit of time in the sequence by increasing TRs and reducing the number of averages. It was not possible to meet the implant manufacturer's $0.1 \mathrm{~W} / \mathrm{kg}$ SAR limit within reasonable scan times by this strategy. Therefore, the $0.1 \mathrm{~W} / \mathrm{kg}$ SAR limit was only reached by additionally reducing the excitation flip angle and the phase resolution (to reduce encoding steps and thus reduce the number of RF pulses). Longerthan-normal scan times of up to 13 minutes were tolerated, given that patients were anesthetized. The SAR level associated with each sequence was determined by examining the scanner log files after running each sequence on a healthy volunteer and subsequently by monitoring the SAR levels recorded in the DICOM header for individual patient scans. Both sequences provided imaging through the ROI from the corpus callosum to the base of the pons.

Our initial tests showed that at $S A R=0.1 \mathrm{~W} / \mathrm{kg}$ and $0.4 \mathrm{~W} / \mathrm{kg}$, the quality of the study was insufficient for stereotaxis. At an SAR of $\sim 0.7 \mathrm{~W} / \mathrm{kg}$, we were able to achieve images adequate for intraoperative stereotaxis, and the images were reviewed by a neuromodulation neurosurgeon (A.Y.M.) and a neuroradiologist (Y.W.L.) for adequacy (Fig 2). Therefore, the following protocol was set up: MPRAGE obtained in the axial plane with the following parameters: $\mathrm{TR}=1870 \mathrm{~ms}, \mathrm{TE}=4 \mathrm{~ms}$, flip angle $=15^{\circ}$, number of signal averages $=1$, FOV $=260 \times 260 \mathrm{~mm}$, matrix size $=256 \times 256$, section thickness $=1.5 \mathrm{~mm}, 176$ sections; a T2-weighted sequence obtained in the axial plane with the following parameters: $\mathrm{TR}=2500 \mathrm{~ms}, \mathrm{TE}=80-83 \mathrm{~ms}$, flip angle $=150^{\circ}$, number of signal averages $=4$, FOV $=260 \times 260 \mathrm{~mm}$, matrix size $=256 \times 256$, section thickness $=2.5 \mathrm{~mm}$, low SAR RF pulse. Using these parameters, we recorded $\sim 1.0 \mathrm{~W} / \mathrm{kg}$ SAR or lower for our test images obtained on an Avanto 1.5T horizontal bore magnet (Siemens, Erlangen, Germany), with a circularly polarized transmit-receive head coil.

Of note, fast spin-echo inversion recovery sequences are routinely used to identify the globus pallidus internus, the primary surgical target used to treat dystonia, but are targeted less frequently than the subthalamic nucleus in Parkinson disease. Preliminary calculations revealed that it would not be possible to modify the sequence to create usable images with acceptable SARs. Surgical targeting for the GPi was thus performed on the T2 images.

\section{Scanning and Patient Monitoring}

Device impedance was checked by personnel from the Center for Neuromodulation before imaging preoperative patients. Patients 


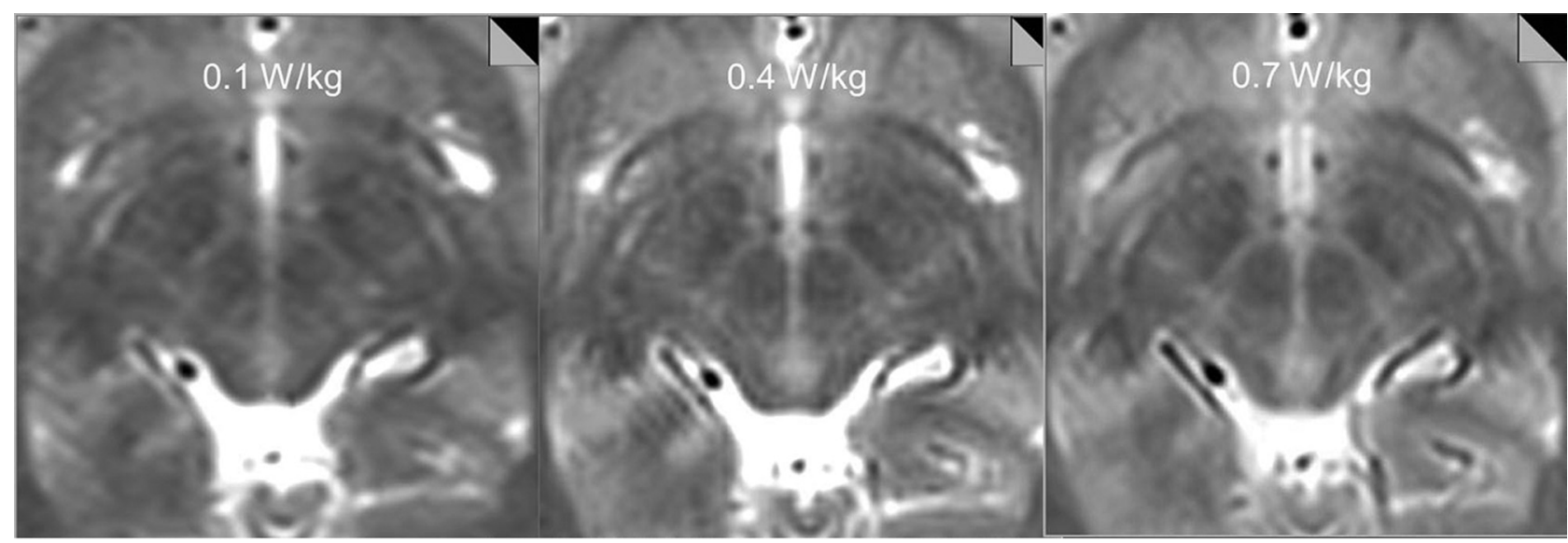

FIG 2. Tests on a volunteer subject show decreasing anatomic detail of the subthalamic region with decreasing SAR. T2-weighted images obtained with an SAR $=0.1 \mathrm{~W} / \mathrm{kg}$ and $0.4 \mathrm{~W} / \mathrm{kg}$ were deemed insufficient for stereotaxis by consensus view between the neuromodulation neurosurgeon and neuroradiologist, while images obtained with an SAR of $0.7 \mathrm{~W} / \mathrm{kg}$ were adequate for intraoperative stereotaxis.

with abnormal impedance readings, broken leads, or electrodes not connected to the pacemaker were excluded because free wires are potentially more hazardous. DBS devices were deactivated before imaging by trained personnel. Imaging was performed by using either an Avanto or Aera 1.5T magnet (Siemens) with circularly polarized transmit-receive head coils, with the patient under general anesthesia to minimize motion in this patient cohort with primary movement disorders. Following imaging, devices were reprogrammed and inspected by trained neuromodulation staff. Patients were strictly monitored during and after MR imaging for complications.

\section{Surgical Technique}

MR imaging scans were all obtained 1-4 weeks before DBS surgery. The day of the operation, a stereotactic headframe (Leksell G frame; Elekta Instruments, Stockholm, Sweden) was affixed to the head with the patient under local anesthesia, and a high-resolution CT scan was performed $(120 \mathrm{kV}, 325 \mathrm{mAs}$, detector configuration of 128 at $0.6-\mathrm{mm}$ collimation). The CT data were then fused via standard stereotactic neurosurgical software (Brainlab, Munich, Germany) to the MR imaging scans for surgical targeting.

\section{Patients}

We retrospectively reviewed the institutional data base of patients who underwent implantation of DBS electrodes between February 1, 2012, and August 1, 2015, by using the optimized low-SAR protocol. Patients who underwent clinical MR imaging performed at our institution with a DBS device in place during imaging were included. No specific exclusion criteria were applied. Chart review was performed to determine outcome, as measured by the rate of subsequent lead revision required relating directly to complications from MR imaging.

\section{Image Analysis}

All MR images were reviewed by a board-certified neuroradiologist (Y.W.L.) and a neurosurgeon specializing in neuromodulation (A.Y.M.). Images were aligned parallel to the anterior/posterior commissure plane. The subthalamic nucleus was assessed on the T2 images on an axial section $4 \mathrm{~mm}$ below the anterior/posterior commissure plane $(\mathrm{z}=-4)$. The globus pallidus was assessed on the axial T2 section containing the anterior/posterior commissures $(z=0)$. Overall image quality was graded as either acceptable or not acceptable for stereotactic surgical guidance on the basis of consensus review for the presurgical patients. The presence of device-related artifacts and any other artifacts was noted, and a comment was made about the impact on image quality. Data were extracted from the DICOM header for each patient scan regarding SAR deposition for each sequence. Comparison of SARs was made between magnets by using the Student $t$ test with a significance level $\alpha=.05$.

\section{RESULTS}

Twenty-nine patients ( 23 male/6 female) were included. The mean age was $58 \pm 14$ years (range, $16-75$ years). All subjects (22 with Parkinson disease, 6 with dystonia, 1 with essential tremor) had neuromodulation implants in situ (Activa PC Models No. 37601 and 37603; Medtronic). There were 25 subthalamic and 4 globus pallidus implants; 10 patients had bilateral electrodes. Of the 29 patients, 5 were scanned on the Aera system and 24, on the Avanto.

Average imaging time was 6 minutes 17 seconds for MPRAGE and 12 minutes 16 seconds for T2. The average SAR deposition for MPRAGE was $0.114 \pm 0.021$ for the Avanto system, $0.090 \pm 0.001$ for the Aera system, and $0.109 \pm 0.021$ overall. The average SAR deposition for the T2-weighted sequence was $1.037 \pm 0.214$ for the Avanto system, $0.828 \pm 0.091$ for the Aera system, and $0.987 \pm 0.210$ overall. SAR depositions for both MPRAGE and T2-weighted sequences were significantly lower on the Aera system ( $P=.012$ and $P=.03$, respectively) (Fig 3 ). Four patients underwent MPRAGE imaging only, for reasons not specified on retrospective review.

All patients tolerated imaging well, with successful completion of MR imaging. No clinical adverse events were reported during or immediately after imaging. All images were deemed by consensus review to be adequate for surgical stereotaxis and were used for subsequent surgical guidance. At an average follow-up time of 553 days $\sim 1.5$ years (range, 2-1251 days; standard deviation, 374 days), there were no adverse patient outcomes and no cases re- 
quiring lead replacement resulting directly from MR imaging complications.

Consensus review of imaging revealed mild device-related local susceptibility artifacts present in all studies that were judged not to affect overall image quality required for subsequent stereotaxis. Mild aliasing artifacts (seen only on T1-weighted images) were present in 6/29 (21\%) cases, and apparent motion artifacts were seen in 4/29 (14\%) cases, all seen on MPRAGE sequences (Fig 4). No such artifacts were present on T2-weighted images.

\section{DISCUSSION}

Our results demonstrate the effective use of an optimized imaging protocol balancing image quality and low SAR to scan patients with DBS implants for surgical planning. The T2-weighted images used applied an average SAR of 0.987 seconds, which, though still higher than the original manufacturer's recommendations, is lower than that published in the literature by using standard sequences and less than one-third the usual SAR when scanning in normal mode. The MPRAGE sequences used in this study were in the range of $0.1 \mathrm{~W} / \mathrm{kg}$ SAR. Low SAR imaging is accomplished with longer imaging times, the use of a low SAR RF pulse, and
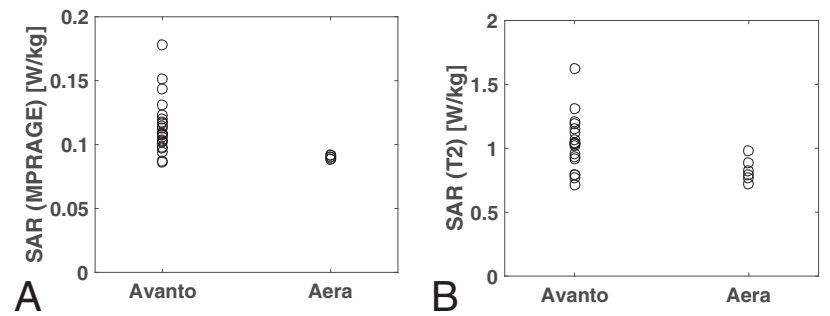

FIG 3. SAR deposition was significantly lower on the Aera system for both MPRAGE $(P=.01)(A)$ and T2-weighted images $(P=.03)(B)$. On the Aera system, all patients were imaged by using an $S A R<1 \mathrm{~W} / \mathrm{kg}$, and the SAR SD was small: \pm 0.02 and $\pm 0.09 \mathrm{~W} / \mathrm{kg}$ for MPRAGE and T2-weighted images, respectively.

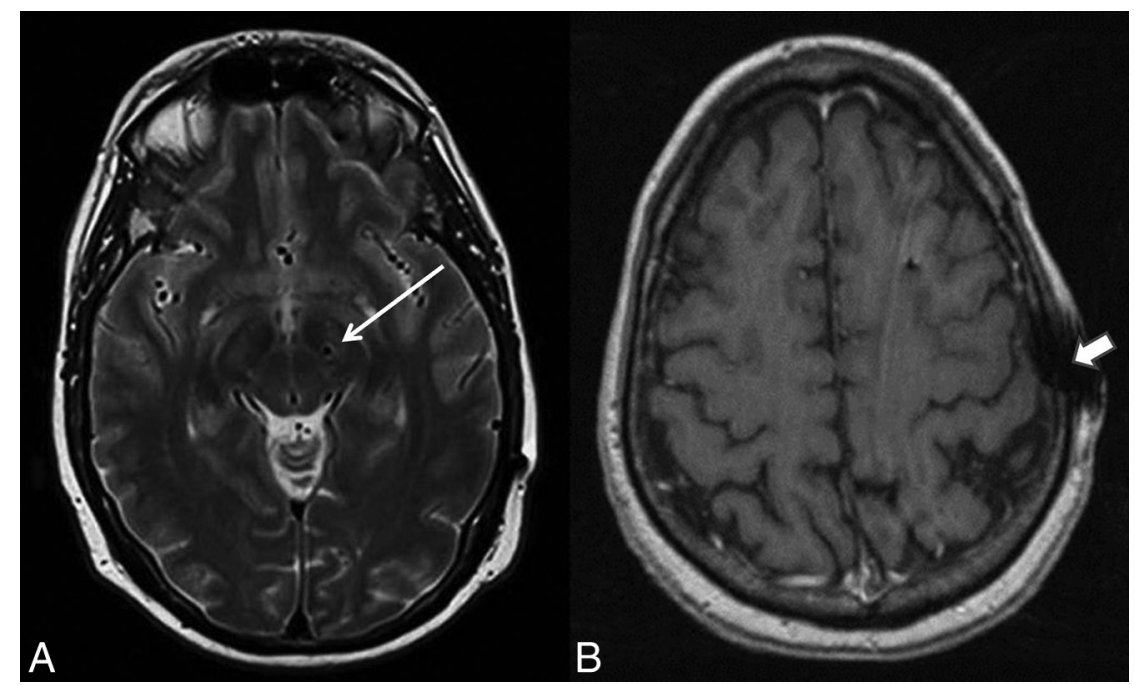

FIG 4. A, Susceptibility from the electrode was very minimal within the adjacent brain parenchyma on T2-weighted images (arrow). B, Device-related local susceptibility in the scalp at the site of electrode entry was seen in most cases on the MPRAGE sequence and was not thought to affect image quality. Additionally, a minority of cases showed artifacts likely attributable to stimulated echoes arising from peripheral fat on MPRAGE images only (arrowhead). Overall, all images were judged to be adequate for presurgical guidance. adjustment of image resolution and was found to be well-tolerated by patients, without complications.

Imaging patients with implantable devices is challenging because of potential safety concerns and artifacts arising from the device, such as local susceptibility effects and spatial distortion. While it is possible to image patients with Medtronic DBS devices, ${ }^{5-7}$ there is limited published information describing appropriate SARs for preoperative planning. Rezai at $\mathrm{al}^{8}$ summarized the major safety concerns of imaging patients with implanted devices, with one of the primary concerns being heating of electrodes due to energy deposition from the transmit RF field. Their in vitro tests showed changes in the temperature of an electrode tip of up to $25.3^{\circ} \mathrm{C}$ by using a transmit/receive body coil in contrast to a maximum change in temperature of $7.1^{\circ} \mathrm{C}$ by using a transmit-receive head coil. Irreversible lesions in brain tissue can occur at temperatures of $45^{\circ} \mathrm{C}$ and higher (ie, $8^{\circ} \mathrm{C}$ above normal body temperature). The degree of electrode heating depends on a number of factors, including routing of the leads, position and orientation of the device, type of coil used, whether leads are connected to the neurostimulator device, where the device is located relative to the isocenter of the MR imaging unit, and energy deposition during imaging or SAR. In the literature, there are 2 reported cases of complications from overheating of DBS electrodes. ${ }^{1,2}$ In the first case, a body coil was used for excitation, and in the other, the patient had free, unconnected leads present. Additional transient adverse events such as dystonia have also been reported. ${ }^{9}$

Due to these potential adverse events, the device manufacturer (Medtronic) issued very conservative guidelines, most notably a head SAR limit of $0.1 \mathrm{~W} / \mathrm{kg}$ (the usual limit is $>30$ times that at $3.2 \mathrm{~W} / \mathrm{kg}$ ). Despite a single report ${ }^{4}$ of 6 subjects in whom quality MR images were obtained by using research sequences that were within the manufacturer's SAR limit, our tests concur with the more widespread experience that this power limitation is insufficient to produce therapeutic-quality T2-weighted images for surgical planning (Fig 2) with FDAapproved sequences. In December 2015, Medtronic issued updated guidelines in which they suggested switching to the use of $\mathrm{B} 1+$ root mean square to calculate RF power with a maximum of 2.0 $\mu \mathrm{T}$, though they continue to recommend that if $\mathrm{B} 1+$ root mean square is not available, the maximum SAR remains $0.1 \mathrm{~W} / \mathrm{kg}$.

Relatively low SAR imaging is accomplished with longer imaging times, use of a low SAR RF pulse, and adjustment of image resolution and is well-tolerated by patients, without complications. We found artifacts to be minimal and more prominent on MPRAGE. Susceptibility effects in the brain parenchyma from the lead itself were minor. Potential room for improvement in image quality may be obtained by shortening the TE (from 104 to below $90 \mathrm{TE}$ ) and reducing the 
bandwidth (from 195 to $160 \mathrm{~Hz} /$ pixel). Limiting section coverage to only the electrode target region would allow more averaging and higher resolution for the same SAR level.

Our results indicate significantly lower SAR for both pulse sequences on the Aera scanner compared with the Avanto scanner with a lower SD of SAR values, which could be due to a variety of factors including coil selection. The 2 magnet systems have a number of differences, including bore diameter, software version, and coil. In our clinical practice, specifications for the Aera and Avanto magnet systems are as follows: D13, $70 \mathrm{~cm}$ diameter bore, circularly polarized send/receive Aera head coil with integrated preamplifier: $315 \times 475 \times 360$ $\mathrm{mm}$ (length $\times$ width $\times$ height) and B17, $60 \mathrm{~cm}$ diameter bore, circularly polarized Avanto head coil with 2 integrated preamplifiers: $480 \times 330 \times 270 \mathrm{~mm}$ (length $\times$ width $\times$ height), respectively. SAR levels reported by different scanners can also vary for the same actual delivered energy, and this has motivated the manufacturer's recent shift to using B1 + root mean square as the safety metric for DBS implants. Different magnets and magnet systems can affect SAR and individualized phantom scanning, and testing should be performed before clinical implementation.

The primary indication for our subjects was presurgical targeting and electrode placement/location confirmation. One limitation of this protocol is that it may not be suitable for other indications such as assessing new or additional pathology. In fact, the protocol does not include whole-brain coverage, which would result in higher SAR. Furthermore, all of our scans were obtained with the patient under general anesthesia, thereby allowing slightly longer scan time without motion. Scan time would certainly be a limitation for awake subjects.

\section{CONCLUSIONS}

Here we introduce a practical, low-SAR MR imaging protocol that can effectively and safely obtain high-quality and high-resolution preoperative images for DBS surgical guidance in patients with a previously implanted electrode. In our multiyear experience with an average T2 head SAR of $0.987 \mathrm{~W} / \mathrm{kg}$, there are no recorded adverse events to date. Optimized coil design, such as the incorporation of a multi-element receive array in a geometry that still allows the use of the stereotactic frame, could improve image quality without increasing the SAR.

Disclosures: Graham C. Wiggins—UNRELATED: Grants/Grants Pending: National Institutes of Health/National Institute of Biomedical Imaging and Bioengineering grant No. P41 EB017183, Comments: The Center for Advanced Imaging Innovation and Research (CAI ${ }^{2}$ R, www.cai2r.net) at New York University School of Medicine is supported by National Institutes of Health/National Institute of Biomedical Imaging and
Bioengineering grant No. P41 EB017183. Alon Y. Mogilner-UNRELATED: Consultancy: Medtronic Neurological; Fees for Participation in Review Activities such as Data Monitoring Boards, Statistical Analysis, Endpoint Committees, and the Like: Medtronic Neurological. Timothy Shepherd-UNRELATED: Grants/Grants Pending: National Institutes of Health National Institute of Aging (NIH 1K23 AG048622-01),* Comments: Alzheimer disease research. This work does not relate to the article in any way; Patents (planned, pending or issued): Velona Technologies, ${ }^{*}$ Comments: I have several recent provisional and full patents. 1) Three of these relate to medical devices for image-guided procedures. I am also in the process of cofounding a start-up company, Velona Technologies, with the goal of bringing these products to market. This work does not relate to the article in any way. 2) An additional patent is related to using multiparametric MRI for evaluating gamma knife radiosurgery treatment responses. This work does not relate to the article in any way; Other: Brainlab contract, * Comments: Through the above patent related to using multiparametric MRI for evaluating gamma knife radiosurgery treatment responses, I am the Principal Investigator on a 2-year industry-funded project ("contract") from Brainlab to study the clinical uses of multiple MRI parameters for predicting and evaluating tumor response to gamma knife therapies. This work does not relate to the article in any way. Yvonne W. Lui-UNRELATED: Grants/Grants Pending: National Institutes of Health R01.* *Money paid to the institution.

\section{REFERENCES}

1. Nutt JG, Anderson VC, Peacock JH, et al. DBS and diathermy interaction induces severe CNS damage. Neurology 2001;56:1384-86 CrossRef Medline

2. Henderson JM, Tkach J, Phillips M, et al. Permanent neurological deficit related to magnetic resonance imaging in a patient with implanted deep brain stimulation electrodes for Parkinson's disease: case report. Neurosurgery 2005;57:E1063; discussion E1063 CrossRef Medline

3. Larson PS, Richardson RM, Starr PA, et al. Magnetic resonance imaging of implanted deep brain stimulators: experience in a large series. Stereotact Funct Neurosurg 2008;86:92-100 Medline

4. Sarkar SN, Papavassiliou E, Hackney DB, et al. Three-dimensional brain MRI for DBS patients within ultra-low radiofrequency power limits. Mov Disord 2014;29:546-49 CrossRef Medline

5. Chhabra V, Sung E, Mewes K, et al. Safety of magnetic resonance imaging of deep brain stimulator systems: a serial imaging and clinical retrospective study. J Neurosurg 2010;112:497-502 CrossRef Medline

6. Fraix V, Chabardes S, Krainik A, et al. Effects of magnetic resonance imaging in patients with implanted deep brain stimulation systems. J Neurosurg 2010;113:1242-45 CrossRef Medline

7. Tagliati M, Jankovic J, Pagan F, et al; National Parkinson Foundation DBS Working Group. Safety of MRI in patients with implanted deep brain stimulation devices. Neuroimage 2009;47(suppl 2):T53-57 CrossRef Medline

8. Rezai AR, Baker KB, Tkach JA, et al. Is magnetic resonance imaging safe for patients with neurostimulation systems used for deep brain stimulation? Neurosurgery 2005;57:1056-62; discussion 1056-62 CrossRef Medline

9. Spiegel J, Fuss G, Backens M, et al. Transient dystonia following magnetic resonance imaging in a patient with deep brain stimulation electrodes for the treatment of Parkinson disease: case report. J Neurosurg 2003;99:772-74 CrossRef Medline 